TRANSACTIONS OF THE

AMERICAN MATHEMATICAL SOCIETY

Volume 348, Number 8, August 1996

\title{
COMPUTATION OF NIELSEN NUMBERS FOR MAPS OF CLOSED SURFACES
}

\author{
O. DAVEY, E. HART, AND K. TRAPP
}

\begin{abstract}
Let $X$ be a closed surface, and let $f: X \rightarrow X$ be a map. We would like to determine $\operatorname{Min}(f):=\min \{|\operatorname{Fix} g|: g \simeq f\}$. Nielsen fixed point theory provides a lower bound $N(f)$ for $\operatorname{Min}(f)$, called the Nielsen number, which is easy to define geometrically and is difficult to compute.

We improve upon an algebraic method of calculating $N(f)$ developed by Fadell and Husseini, so that the method becomes algorithmic for orientable closed surfaces up to the distinguishing of Reidemeister orbits. Our improvement makes tractable calculations of Nielsen numbers for many maps on surfaces of negative Euler characteristic. We apply the improved method to selfmaps on the connected sum of two tori including classes of examples for which no other method is known. We also include the application of this algebraic method to maps on the Klein bottle $K$. Nielsen numbers for maps on $K$ were first calculated (geometrically) by Halpern. We include a sketch of Halpern's never published proof that $N(f)=\operatorname{Min}(f)$ for all maps $f$ on $K$.
\end{abstract}

\section{INTRODUCTION}

Let $X$ be a closed surface, and let $f: X \rightarrow X$ be a map. We would like to know the minimum number of fixed points of all maps that are homotopic to $f$. This minimum number is given by

$$
\operatorname{Min}(f)=\min \{|\operatorname{Fix} g|: g \simeq f\} .
$$

Nielsen fixed point theory provides a lower bound for $\operatorname{Min}(f)$ that is called the Nielsen number $N(f)$. The Nielsen number is easy to define geometrically, but it is difficult to compute. We improve upon an algebraic method of computing $N(f)$ that was developed by Fadell and Husseini in [8] so that, for orientable closed surfaces, all but one step is algorithmic, and we apply the improved method to self-maps on the connected sum of two tori (the double torus) and to self-maps on the Klein bottle. While other methods are available for some of our calculations, the method we use is completely different, and we use it to find previously unknown Nielsen numbers for classes of maps on the double torus for which no other method is available.

To define $N(f)$, we first partition $\operatorname{Fix}(f)$ into equivalence classes with two fixed points $x$ and $y$ equivalent if and only if there exists a path $\alpha$ from $x$ to $y$ with $f \circ \alpha$ path homotopic to $\alpha$. Each equivalence class is assigned an index, which is an

Received by the editors October 3, 1995.

1991 Mathematics Subject Classification. Primary 55M20, 57M20; Secondary 57M05.

Key words and phrases. Nielsen fixed point theory, Topological fixed point theory, Lefschetz number.

The authors were partially supported by NSF grant \#DMS9322328.

(C)1996 American Mathematical Society 
integer that is a generalization of the multiplicity of a zero of a complex analytic function. The Nielsen number of $f$ is the number of classes of fixed points with non-zero index, and it is a homotopy invariant. The Lefschetz number $L(f)$ is the sum of the indices. For background on Nielsen fixed point theory, see [5], [15] and [19].

Nielsen defined fixed point classes and the Nielsen number in the restricted setting of self-homeomorphisms of surfaces in [24] in 1927. The study of the Nielsen number and the minimum number for self-maps (not only homeomorphisms) on closed surfaces continues to present challenges. In most cases, the value of $\operatorname{Min}(f)$ is unknown and the value of $N(f)$ is difficult to calculate or is currently unknown. Jiang includes a set of interesting historical notes in his book [15].

The Nielsen number for self-maps on the sphere $S^{2}$ provides no more information than the Lefschetz number, because any two fixed points are Nielsen equivalent. But the situation for other surfaces is much more complicated. The Nielsen number for any map on the torus was calculated in the 1920's by Nielsen and Brouwer in [23] and [4] and independently in 1975 by Brooks, Brown, Pak and Taylor in [3]. Results for the projective plane have been known since 1927, see [13].

The method used here was developed by Fadell and Husseini in [8]. For $\pi$ the fundamental group of a compact surface $X$, the method involves using the Fox calculus (see [7]) to calculate algebraic traces of homomorphisms on the cellular chain groups for the universal cover of $X$. These homomorphisms are induced by a lift of $f$ to the covering space, and the traces are elements of the group ring $\mathbb{Z}[\pi]$. In Section 2 we provide the details. The Nielsen number of $f$ is obtained by a sequence of steps that is almost an algorithm. There are two steps that are not algorithmic. The first is the calculation of the trace in dimension two, and we improve upon this step so that it is algorithmic for orientable closed surfaces. This result is in Section 3 and is described below. The second step for which there is no algorithm is the distinguishing of Reidemeister orbits. This is related to the conjugacy problem in combinatorial group theory. While there are several methods for distinguishing orbits, there is no guarantee that they will work for a given example. The computer algebra system Magma, [2], has been used to perform a search for elements of limited word-length in a given Reidemeister orbit for Example 4 of Section 4.

Our main result is in Section 3, where we develop an algorithm for determining the algebraic trace in dimension 2. This new result has made calculations of Nielsen numbers for maps on the double torus much more manageable. Examples are contained in Section 4. This improvement will be useful for the calculation of Nielsen numbers for other surfaces with negative Euler characteristic.

In Section 4 we use the method of Fadell and Husseini for the calculation of the Nielsen numbers for some classes of self-maps on the double torus $T \# T$. Because the fundamental group of $T \# T$ is so difficult to work with, the main result in Section 3 is crucial in making the calculations possible. We include several classes of examples including two that cannot be done by any other method. Even when other methods could be employed, the algebraic method employed here is attractive in its relative simplicity as long as the Reidemeister orbits can be distinguished. Other methods for calculating $N(f)$ for self-maps on the double torus are the following: For maps that are homeomorphisms, Kelly has an algorithm in [18] that involves geometric homotopy theory. See also the work of Bestvina and Handel in [1] and the work of Franks and Misiurewicz in [9] for geometric algorithms. For maps that are not homeomorphisms, there are two situations in which other methods apply. 
Maps that factor through $S^{1}$ have Nielsen numbers that are easy to calculate. The Nielsen numbers for certain of the maps that factor through the figure-eight can be calculated using a method of Wagner [25]. We include a class of homomorphisms for which none of these approaches applies. For these homomorphisms, the FadellHusseini method is the only known method for calculating the Nielsen number.

Next we turn to the Klein bottle. The first calculation of Nielsen numbers for all maps on the Klein bottle $K$ was in 1978 by Halpern in [10]. A method using fibrations for calculating Nielsen numbers of self-maps on the Klein bottle has been found by Heath, Keppelmann and Wong [12]. McCord has results that prove that the Nielsen numbers on the Klein bottle can be calculated in terms of determinants. See [21] and [22].

In Section 5 we use the Fadell-Husseini method, which is completely different from the methods just mentioned, to determine $N(f)$ for any map $f$ on $K$ in terms of the homomorphism induced by $f$ on the fundamental group. Halpern's method was geometric, and the algebraic approach given here is much cleaner in that it is almost algorithmic. These algebraic calculations for the Klein bottle first appeared in Hart's doctoral dissertation [11].

We have addressed questions involving the calculation of Nielsen numbers. Another question of interest is: How accurately does the Nielsen number reflect the minimum number? Recall that a closed surface is called Wecken in [6] if for every self-map $f$ of the surface we have $N(f)=\operatorname{Min}(f)$. Brown summarizes results involving the Wecken property for surfaces and other manifolds in [6]. Kelly [17] and Jiang [16] proved that surfaces with negative Euler characteristic are totally nonWecken. That is, for any $n \geq 1$ there exists a self-map $f$ of the surface for which $\operatorname{Min}(f)-N(f)=n$. Thus for self-maps on $T \# T$ that are not homeomorphisms, the Nielsen number could be an arbitrarily bad estimate of the minimum number. But at least the Nielsen number is a lower bound.

The algebraic methods used here for the Klein bottle have one disadvantage when compared with Halpern's geometric approach. Halpern was able to prove that $K$ is Wecken by exhibiting a map $g$ for each homotopy class that has $\operatorname{Min}(g)=$ $N(g)$. Because Halpern's paper was never published, we include as the last part of Section 5 a sketch of his proof of the Wecken property for $K$.

To summarize, the paper is organized as follows. In Section 2 we provide the needed background and describe the Fadell-Husseini method. Section 3 contains our main result, which simplifies the Fadell-Husseini method for surfaces with negative Euler characteristic. We apply the improved method in Section 4 to self-maps of $T \# T$ and include classes of examples for which there is no other method for calculating $N(f)$. Section 5 contains calculations of Nielsen numbers for all self-maps of the Klein bottle using the Fadell-Husseini method. We also include Halpern's proof (from the unpublished paper [10]) of the Wecken property for the Klein bottle. ${ }^{1}$

The main result in Section 3 was inspired by the work of Davey and Trapp, who were undergraduates working under the guidance of Hart as part of an NSFfunded REU program at Hope College during the summer of 1994. Davey and Trapp calculated the Nielsen number for numerous examples of self-maps on $T \# T$ without the advantage of the formula from Section 3. They were searching for a pattern for the trace in dimension two when their eight-week program ended. Thanks are due to Helga Schirmer and to Robert F. Brown for their helpful comments on drafts of

\footnotetext{
${ }^{1}$ In 8.3 of [12], it is proven that all solvmanifolds (hence the Klein bottle) are Wecken.
} 
this paper and to Edward Fadell, who served as the advisor for Hart's dissertation in 1991. Edward Keppelmann provided the programming for the example using the software package Magma [2].

\section{Preliminaries}

Let $X$ be a closed, non-simply connected surface with base point $x_{0}$, and let $\pi=\pi_{1}\left(X, x_{0}\right)$. The universal covering space of $X$ is denoted by $\widetilde{X}$, with covering projection $\eta: \widetilde{X} \rightarrow X$. We identify $X$ with the usual CW-structure on $X$ given by the 0 -cell $x_{0}$, a single 2-cell, and the usual 1 -cells. For any map $f: X \rightarrow X$, there is a cellular approximation to $f$ with the same Nielsen number as $f$. Thus we may assume $f$ is cellular and fixes $x_{0}$. The CW-structure on $X$ induces a CW-structure on $\widetilde{X}$. We must choose a base point of $\widetilde{X}$ that covers $x_{0}$.

For every homomorphism $\phi: \pi \rightarrow \pi$ there is a cellular map that induces $\phi$, because $X$ is a $K(\pi, 1)$, so we concentrate on the homomorphisms rather than the maps. Let $\phi: \pi \rightarrow \pi$ and let $f$ be a cellular map that induces $\phi$. We may choose a lift $\widetilde{f}: \widetilde{X} \rightarrow \widetilde{X}$ of $f$ so that $\widetilde{f}$ is also cellular and fixes the base point in $\widetilde{X}$. Let $C_{q}(\widetilde{X})$ be the group of cellular $q$-chains over $\mathbb{Z}$. Then $\widetilde{f}$ induces the homomorphism $\widetilde{f}_{q}: C_{q}(\widetilde{X}) \rightarrow C_{q}(\widetilde{X})$. The group $C_{q}(\widetilde{X})$ is a free, finitely generated $\mathbb{Z}[\pi]-$ module, and $\widetilde{f}_{q}$ is almost a $\mathbb{Z}[\pi]$-module homomorphism. Specifically, $\widetilde{f}_{q}$ respects the $\mathbb{Z}[\pi]-$ structure in the following sense. For all $\sigma \in \pi$ and for all $u \in C_{q}(\widetilde{X}), \widetilde{f}_{q}(\sigma u)=$ $\phi(\sigma) \widetilde{f}_{q}(u)$. Let $M_{q}$ denote the matrix for $\widetilde{f}_{q}$ over $\mathbb{Z}[\pi]$.

The homomorphism $\phi$ induces a left group action of $\pi$ on $\pi$, called the Reidemeister action, given by $\sigma \cdot \alpha=\phi(\sigma) \alpha \sigma^{-1}$ for all $\alpha, \sigma \in \pi$. The orbit of $\alpha$ under this action is called the Reidemeister orbit, and the set of Reidemeister orbits is denoted by $R(\phi)$. Let $\rho: \pi \rightarrow R(\phi)$ be the function that sends each element of $\pi$ to its orbit, and extend $\rho$ linearly to a function $\rho: \mathbb{Z}[\pi] \rightarrow \mathbb{Z}(R(\phi))$. Here $\mathbb{Z}(R(\phi))$ is the free $\mathbb{Z}$-module generated by the set $R(\phi)$.

The generalized Lefschetz number is defined to be

$$
L(f, \tilde{f})=\sum_{q}(-1)^{q} \rho\left(\operatorname{tr} M_{q}\right) \in \mathbb{Z}(R(\phi)) .
$$

As Husseini proves in [14], for $X$ a finite CW complex we have

$$
L(f, \tilde{f})=\sum_{\alpha \in W} i([\alpha])[\alpha]
$$

where $W$ is a transversal for $R(\phi)$ and $i([\alpha])$ is the fixed point index of the Nielsen class $\eta \operatorname{Fix}\left(\alpha^{-1} \widetilde{f}\right)$. This second formula for $L(f, \widetilde{f})$ is reduced in the sense that each orbit occurs at most once. When $L(f, \widetilde{f})$ has been reduced to this form (not an easy task, and no algorithm is available), then $N(f)$ is the number of terms with non-zero coefficient in $L(f, \widetilde{f})$. The problem of distinguishing orbits can be a serious stumbling block. It is related to the conjugacy problem for finitely generated groups.

The method of Fadell and Husseini for closed surfaces. Fadell and Husseini provide in [8] a method for calculating $L(f, \widetilde{f})$ in terms of $\phi$ using the Fox calculus developed in [7]. For a closed surface we have $\pi$ given by $\left\langle x_{1}, \ldots, x_{k} ; R\right\rangle$, with $R$ a relator in the generators $x_{1}, \ldots, x_{k}$ determined by the cellular structure of $X$. Let $F$ be the free group generated by $x_{1}, \ldots, x_{k}$, and let $\phi_{F}: F \rightarrow F$ be the 
homomorphism that takes $x_{i}$ to the word in $F$ given by $\phi\left(x_{i}\right)$ for each $i=1, \ldots, k$. There are many ways to represent a given homomorphism $\phi$, and each would give a different homomorphism $\phi_{F}$. We will find this fact useful in simplifying the calculation of Nielsen numbers on the double torus in Section 4.

The Fox calculus (see [7]) provides a formula for describing the lift of a loop $\alpha$ in $X$ to a path in $\widetilde{X}$ in terms of the element of $F$ that corresponds to $\alpha$. For each generator $x_{i}, i=1, \ldots, k$, the Fox partial derivative operator is a function $\partial / \partial x_{i}: \mathbb{Z}[F] \rightarrow \mathbb{Z}[F]$. Here we always immediately project the output of this function into $\mathbb{Z}[\pi]$. The Fox partial derivative operator has the following basic properties:

1. For $u, v \in F, \frac{\partial}{\partial x_{i}}(u v)=\frac{\partial u}{\partial x_{i}}+u \frac{\partial v}{\partial x_{i}}$,

2. $\frac{\partial x_{i}}{\partial x_{j}}=\delta_{i j}$, and $\frac{\partial 1}{\partial x_{i}}=0$.

These properties imply that

3. $\frac{\partial x_{i}^{k}}{\partial x_{i}}=1+x_{i}+\ldots+x_{i}^{k-1}$ for $k \geq 1$,

4. $\frac{\partial x_{i}^{-k}}{\partial x_{i}}=-\left(x_{i}^{-1}+x_{i}^{-2}+\ldots+x_{i}^{-k}\right)$ for $k \geq 1$,

5. $\left(1-x_{i}\right) \frac{\partial x_{i}^{k}}{\partial x_{i}}=1-x_{i}^{k}$ for all $k$,

6. for any $w \in F, \frac{\partial w^{-1}}{\partial x_{i}}=-w^{-1} \frac{\partial w}{\partial x_{i}}$.

Let $J\left(\phi_{F}\right)$ be the Jacobian matrix $\left[\partial \phi_{F}\left(x_{i}\right) / \partial x_{j}\right]$, which is the matrix for $\widetilde{f}_{1}$ over $\mathbb{Z}[\pi]$. For any element $w$ in $F$, let the gradient of $w$ be given by the vector

$$
\nabla w=\left(\frac{\partial w}{\partial x_{1}}, \ldots, \frac{\partial w}{\partial x_{k}}\right) .
$$

Let $\widetilde{u}$ be the 2 -cell in $\widetilde{X}$ that generates $C_{2}(\widetilde{X})$ as a $\mathbb{Z}[\pi]-$ module. Then $\widetilde{f}(\widetilde{u})=A \widetilde{u}$ for some element $\mathrm{A}$ of $\mathbb{Z}[\pi]$. The formula for the generalized Lefschetz number developed by Fadell and Husseini in [8] is

$$
L(f, \widetilde{f})=\rho\left(1-\operatorname{tr} J\left(\phi_{F}\right)+A\right),
$$

with $A$ the unique element of $\mathbb{Z}[\pi]$ satisfying $A(\nabla R)=\phi(\nabla R) J\left(\phi_{F}\right)$. The uniqueness of $A$ follows from the fact that $X$ is aspherical and thus the boundary map $\partial: C_{2}(\widetilde{X}) \rightarrow C_{1}(\widetilde{X})$ is injective. Also, $\phi(\nabla R)$ makes sense when we extend $\phi$ linearly to $\phi: \mathbb{Z}[\pi] \rightarrow \mathbb{Z}[\pi]$ and then apply $\phi$ to each coordinate of $\nabla R$.

There are two steps in this method for which there are no algorithms. First, finding the element $A$ of $\mathbb{Z}[\pi]$ is challenging, with no method available that will always work. In Section 3 we develop an algorithm for calculating $A$ that makes calculations simpler for closed surfaces with negative Euler characteristic. Second, the Reidemeister orbits appearing in the $\operatorname{sum} L(f, \widetilde{f})$ must be distinguished. There are several methods for attacking this problem, but none is certain to provide an answer.

Two techniques for distinguishing Reidemeister orbits that are mentioned in [8] are the following. The fact that $\rho(x)=\rho(\phi(x))$ for all $x \in \pi$ can be quite useful. By iterating $\phi$ we can prove that many elements are in the same orbit. Also, if $N$ is a normal subgroup of $\pi$ for which $\phi(N) \subseteq N$, we can consider the induced action on $\bar{\pi}=\pi / N$. If two elements $x, y \in \pi$ have images $\bar{x}$ and $\bar{y}$ in $\bar{\pi}$ that are in distinct 
orbits of the induced action, then we know that $\rho(x)$ and $\rho(y)$ are also distinct. This is particularly useful when $\bar{\pi}$ is the abelianization of $\pi$. In Example 4.4 of Section 4 , we find two orbits that cannot be distinguished by either of these methods. We describe the use of the computer algebra system Magma [2] to aid our intuition for this example.

\section{Calculation of the trace in Dimension two}

One difficulty in calculating the generalized Lefschetz number using the method described in Section 2 is determining the value of the trace $A$ in dimension two. One must solve for $A$ in the formula

$$
A(\nabla R)=\phi(\nabla R) J\left(\phi_{F}\right)
$$

and no algorithm is available. Here we develop such an algorithm for orientable closed surfaces. We first use Dehn's algorithm, see pp. 246-252 of [20], to write $\phi_{F}(R)$ as a product of conjugates of $R$ and $R^{-1}$. Once $\phi_{F}(R)$ is in this form, then $A$ can be computed easily using the theorem below. With this new result, $\phi(\nabla R)$ and the off-diagonal entries of $J\left(\phi_{F}\right)$ no longer need to be computed when calculating $L(f, \tilde{f})$. For the double torus $T \# T$, every homomorphism we have examined has a representation $\phi_{F}$ for which it is very easy to write $\phi_{F}(R)$ in the necessary form. The fact that $\phi$ must take $R$ to 1 in $\pi$ limits the possible forms for $\phi_{F}(R)$.

Because $\phi_{F}(R)$ is an element of the normal closure $N$ of the subgroup $\langle R\rangle$ in $F$, we can write $\phi_{F}(R)=\prod_{i=1}^{n} y_{i} R^{\lambda_{i}} y_{i}^{-1}$ for some $n \in \mathbb{Z}^{+}$, with $\lambda_{i} \in \mathbb{Z}$ and $y_{i} \in F$ for each $i$. This expression for $\phi_{F}(R)$ is not unique, and it is not necessarily reduced as a word in $F$. When $\phi$ is an isomorphism, the element $\phi_{F}(R)$ must be a conjugate of $R$ or $R^{-1}$ (see page 49 of [20]), and then the following theorem implies that $A$ will be a monomial in $\mathbb{Z}[\pi]$.

Theorem 3.1. For $X$ any closed surface and $\phi: \pi \rightarrow \pi$ any homomorphism on the fundamental group of $X$, we have the following formula for $A$. Let $\phi_{F}(R)=$ $\prod_{i=1}^{n} y_{i} R^{\lambda_{i}} y_{i}^{-1}$, with $\lambda_{i} \in \mathbb{Z}$ and $y_{i} \in F$ for each $i$. Then $A=\sum_{i} \lambda_{i} y_{i} \in \mathbb{Z}[\pi]$. The expression for $\phi_{F}(R)$ as an element of $N$ is not unique, but the resulting value for $A$ as an element of $\mathbb{Z}[\pi]$ is independent of the expression chosen for $\phi_{F}(R)$.

Proof. In Step 1 we will prove that any solution $A$ to equation (1) must also satisfy $A(\nabla R)=\nabla\left(\phi_{F}(R)\right)$. In Step 2 we will prove that for any generator $x_{j}$ of $F$,

$$
\frac{\partial \prod_{i=1}^{n} y_{i} R^{\lambda_{i}} y_{i}^{-1}}{\partial x_{j}}=\sum_{i=1}^{n} \lambda_{i} y_{i} \frac{\partial R}{\partial x_{j}} .
$$

Once this equality is proven, we will have $\nabla\left(\phi_{F}(R)\right)=\left(\sum_{i=1}^{n} \lambda_{i} y_{i}\right)(\nabla R)$. So for any expression of $\phi_{F}(R)$ of the form $\prod_{i=1}^{n} y_{i} R^{\lambda_{i}} y_{i}^{-1}$, we will have $A=\left(\sum_{i=1}^{n} \lambda_{i} y_{i}\right)$. Because 
$A$ is the unique element of $\mathbb{Z}[\pi]$ that satisfies equation (1), we know that this sum is independent of the way in which $\phi_{F}(R)$ is expressed.

Step 1 . We prove that for any $w \in F$ we have $\phi(\nabla w) J\left(\phi_{F}\right)=\nabla\left(\phi_{F}(w)\right)$, so that $A(\nabla R)=\nabla\left(\phi_{F}(R)\right)$.

Let $\tilde{X}$ be the universal cover of $X$. Let $\vec{x}$ be the column vector with each coordinate consisting of a lift to $\widetilde{X}$ for each of the generators of $\pi$. Then $\vec{x}$ is a vector of basis elements for $C_{1}(\tilde{X})$. For every $w \in F$, let $\omega$ be the corresponding path in $X$. The element $(\nabla w) \vec{x} \in C_{1}(\widetilde{X})$ is the chain that represents the lift of $\omega$ to a path $\widetilde{\omega}$ that begins at the base point in $\widetilde{X}$. Thus $\nabla\left(\phi_{F}(R)\right) \vec{x}$ represents the lift of the path corresponding to $\phi_{F}(R)$.

For the covering projection $\eta: \widetilde{X} \rightarrow X$, we have $\eta \tilde{f}=f \eta$. Thus $\widetilde{f}(\widetilde{\omega})$ and $\widetilde{(f \omega)}$ are both lifts of $f \omega$ that begin at the base point. So the corresponding elements of $C_{1}(\tilde{X})$ must be equal. This implies that $\tilde{f}_{1}((\nabla w) \vec{x})=\left(\nabla\left(\phi_{F}(w)\right) \vec{x}\right.$, and

$$
\widetilde{f}_{1}((\nabla w) \vec{x})=\phi(\nabla w)\left(\widetilde{f}_{1} \vec{x}\right)=\phi(\nabla w) J\left(\phi_{F}\right) \vec{x} .
$$

Thus $\phi(\nabla w) J\left(\phi_{F}\right)=\left(\nabla\left(\phi_{F}(w)\right)\right.$ for any $w \in F$. We set $w$ equal to $R$ and conclude that $A$ is the unique element of $\mathbb{Z}[\pi]$ that satisfies $A(\nabla R)=\nabla\left(\phi_{F}(R)\right)$. Step 1 is proven.

Step 2. We prove that whenever $\phi_{F}(R)$ is expressed in the form

$$
\phi_{F}(R)=\prod_{i=1}^{n} y_{i} R^{\lambda_{i}} y_{i}^{-1}
$$

as in the statement of the theorem, then for any generator $x_{j}$ of $F$ we have

$$
\frac{\partial \phi_{F}(R)}{\partial x_{j}}=\sum_{i=1}^{n} \lambda_{i} y_{i} \frac{\partial R}{\partial x_{j}}
$$

It is sufficient to prove Step 2 in the case for which $\lambda_{i} \in\{1,-1,0\}$ for all $i$, because $y_{i} R^{\lambda_{i}} y_{i}^{-1}$ can be written as either $\left(y_{i} R y_{i}^{-1}\right)^{\lambda_{i}}$ or $\left(y_{i} R^{-1} y_{i}^{-1}\right)^{\lambda_{i}}$. So we assume that for each $i$ we have $\lambda_{i} \in\{1,-1,0\}$, but we note that this expression of $\phi_{F}(R)$ is not necessarily reduced in $F$.

First, for some $i$ consider $\frac{\partial y_{i} R^{\lambda_{i}} y_{i}^{-1}}{\partial x_{j}}$. We have

$$
\frac{\partial y_{i} R^{\lambda_{i}} y_{i}^{-1}}{\partial x_{j}}=\frac{\partial y_{i}}{\partial x_{j}}+y_{i} \frac{\partial R^{\lambda_{i}}}{\partial x_{j}}+y_{i} R^{\lambda_{i}} \frac{\partial y_{i}^{-1}}{\partial x_{j}} .
$$

We may use Property 6 of the Fox calculus and the fact that the coefficients are elements of $\pi$ to write, for $\lambda_{i} \in\{1,-1,0\}$,

$$
\begin{gathered}
\frac{\partial y_{i} R^{\lambda_{i}} y_{i}^{-1}}{\partial x_{j}}=\frac{\partial y_{i}}{\partial x_{j}}+\lambda_{i} y_{i} R^{\lambda_{i}} \frac{\partial R}{\partial x_{j}}-y_{i} R^{\lambda_{i}} y_{i}^{-1} \frac{\partial y_{i}}{\partial x_{j}} \\
=\frac{\partial y_{i}}{\partial x_{j}}+\lambda_{i} y_{i} \frac{\partial R}{\partial x_{j}}-\frac{\partial y_{i}}{\partial x_{j}}=\lambda_{i} y_{i} \frac{\partial R}{\partial x_{j}} .
\end{gathered}
$$

We complete the proof of Step 2 by inducting on $n$. We have 


$$
\begin{gathered}
\frac{\partial \prod_{i=1}^{n} y_{i} R^{\lambda_{i}} y_{i}^{-1}}{\partial x_{j}}=\frac{\partial\left[\left(y_{1} R^{\lambda_{1}} y_{1}^{-1}\right)\left(\prod_{i=2}^{n} y_{i} R^{\lambda_{i}} y_{i}^{-1}\right)\right]}{\partial x_{j}} \\
=\frac{\partial\left(y_{1} R^{\lambda_{1}} y_{1}^{-1}\right)}{\partial x_{j}}+\left(y_{1} R^{\lambda_{1}} y_{1}^{-1}\right)\left(\sum_{i=2}^{n} \lambda_{i} y_{i}\right) \frac{\partial R}{\partial x_{j}} .
\end{gathered}
$$

When the coefficients are viewed as elements of $\pi$, we have

$$
\frac{\partial \prod_{i=1}^{n} y_{i} R^{\lambda_{i}} y_{i}^{-1}}{\partial x_{j}}=\lambda_{1} y_{1} \frac{\partial R}{\partial x_{j}}+\left(\sum_{i=2}^{n} \lambda_{i} y_{i}\right) \frac{\partial R}{\partial x_{j}}=\left(\sum_{i=1}^{n} \lambda_{i} y_{i}\right) \frac{\partial R}{\partial x_{j}} .
$$

Thus

$$
\nabla\left(\phi_{F}(R)\right)=\left(\sum_{i=1}^{n} \lambda_{i} y_{i}\right) \nabla R
$$

\section{EXAMPles FOR THE DOUBle TORUS}

In this section we make use of the Fadell-Husseini method and our new result from Section 3 to compute Nielsen numbers of self-maps on the double torus. We include a class of examples that cannot be computed by any other known process. In Example 4.1, we demonstrate that the new formula from Section 3 is a significant improvement to the Fadell-Husseini method. As we demonstrate in Example 4.2, a judicious choice of $\phi_{F}$ for a given $\phi$ can simplify the calculations dramatically. For Examples 4.6 and 4.7, the Fadell-Husseini method is the only known method for calculating the Nielsen number. Even when other methods are available, this method provides an algebraic approach that is quite tractable as long as the Reidemeister orbits can be distinguished.

The advantage of the new formula for $A$. To demonstrate the advantage of the new formula for $A$, in the first example we calculate $A$ both with and also without the new formula. For all the other examples of this section, we use the new formula only. The maps $f$ in Example 4.1, Example 4.3, and Example 4.4 are homeomorphisms, so there are other methods available for finding $N(f)$. When $f$ is a homeomorphism, we have $N(f)$ equal to $\operatorname{Min}(f)$, so the more geometric methods of [18], [9], and [1] apply. The method used here is quite different, being entirely algebraic. The fundamental group of $T \# T$ is denoted by

$$
\pi=\left\langle a, b, c, d: a b a^{-1} b^{-1} c d c^{-1} d^{-1}\right\rangle .
$$

Example 4.1. Let $n \geq 2$ and let $\phi$ be given by

$$
\begin{aligned}
& a \rightarrow b^{-n+1} a^{-1}, \quad c \rightarrow d, \\
& b \rightarrow a b^{n}, \quad d \rightarrow c \text {. }
\end{aligned}
$$

We claim that $N(f)=n-1$.

First we calculate $A$ without the formula from Section 3. We have

$$
\nabla R=\left(1-a b a^{-1}, a-a b a^{-1} b^{-1}, a b a^{-1} b^{-1}-d, d c d^{-1}-1\right),
$$

and thus

$$
\phi(\nabla R)=\left(1-b a b^{n-1}, b^{-n+1} a^{-1}-b a b^{-1} a^{-1}, b a b^{-1} a^{-1}-c, c d c^{-1}-1\right) .
$$


The Jacobian $J\left(\phi_{F}\right)$ is

$$
\left[\begin{array}{cccc}
-b^{-n+1} a^{-1} & -\sum_{i=1}^{n-1} b^{-i} & 0 & 0 \\
1 & a \sum_{i=0}^{n-1} b^{i} & 0 & 0 \\
0 & 0 & 0 & 1 \\
0 & 0 & 1 & 0
\end{array}\right]
$$

We must find the unique element $A \in \mathbb{Z}[\pi]$ that satisfies

$$
A \nabla R=\phi(\nabla R) J\left(\phi_{F}\right) .
$$

By an argument similar to that of Proposition 5.2 below, we need only find a value for $A$ that satisfies one coordinate of the above equation. The fourth coordinate appears simple, so we seek a solution to

$$
A\left(d c d^{-1}-1\right)=\left(b a b^{-1} a^{-1}-c\right) .
$$

There is no algorithm for this step, and one must observe that $A=-b a b^{-1} a^{-1}$ is a solution.

Next we repeat the calculation of $A$ using the formula of Section 3. We see that $\phi_{F}(R)=b a b^{-1} a^{-1} d c d^{-1} c^{-1}$. This word in $F$ closely resembles $R$, and it is in fact a cyclic permutation of the letters in the word $R^{-1}$. Thus $\phi_{F}(R)=$ $b a b^{-1} a^{-1} R^{-1} a b a^{-1} b^{-1}$, and $A=-b a b^{-1} a^{-1}$. We never need to calculate $\nabla R$, $\phi(\nabla R)$, nor the off-diagonal entries in the Jacobian.

To continue the calculation of $N(f)$, we formulate the generalized Lefschetz number using the diagonal entries of the Jacobian:

$$
L(f, \widetilde{f})=\rho\left(1+b^{-n+1} a^{-1}-\sum_{i=0}^{n-1} a b^{i}-b a b^{-1} a^{-1}\right) .
$$

We reduce $L(f, \tilde{f})$ by noting that $\rho(a)=\rho(\phi(a))=\rho\left(b^{-n+1} a^{-1}\right)$. Thus the second and third terms in the original expression for $L(f, \tilde{f})$ cancel. It can be shown, using the abelianization of $\pi$, that $\rho(a b), \ldots, \rho\left(a b^{n-1}\right)$ are all distinct and that $\rho(a b), \ldots, \rho\left(a b^{n-2}\right)$ are all distinct from $\rho(1)$ and $\rho\left(b a b^{-1} a^{-1}\right)$. It remains to determine whether any two of $\rho(1), \rho\left(a b^{n-1}\right)$, and $\rho\left(b a b^{-1} a^{-1}\right)$ are equal. Here we reach the second part of the Fadell-Husseini method for which there is no algorithm. We note that $\rho(1)=\rho\left(\phi(d c)(d c)^{-1}\right)=\rho\left(c d c^{-1} d^{-1}\right)=\rho\left(b a b^{-1} a^{-1}\right)$. Thus the reduced form of $L(f, \widetilde{f})$ is given by

$$
L(f, \widetilde{f})=\rho\left(-\sum_{i=1}^{n-1} a b^{i}\right),
$$

and $N(f)=n-1$.

The importance of the choice of $\phi_{F}$. For any homomorphism $\phi: \pi \rightarrow \pi$, there are many possible forms in which to write the image of the generators of $\pi$ under $\phi$. By choosing a form for $\phi$ that makes $\phi_{F}(R)$ as simple as possible, we simplify the calculation of $N(f)$ significantly. Recall that $A$ and $L(f, \tilde{f})$ depend on the choice of $\phi_{F}$, but the Nielsen number does not. To demonstrate the importance of the choice of $\phi_{F}$, we compute $N(f)$ for two choices of $\phi_{F}$ in the following example. 
This example could be done by factoring $f$ through $S^{1}$, but its simplicity makes our point without requiring an undesirable amount of calculation.

Example 4.2. Consider the homomorphism $\phi$ given by

$$
\begin{aligned}
& a \rightarrow c d c^{-1} d^{-1}, \quad c \rightarrow 1, \\
& b \rightarrow a b a^{-1} b^{-1}, \quad d \rightarrow 1 .
\end{aligned}
$$

This homomorphism can also be expressed by

$$
\begin{aligned}
& a \rightarrow c d c^{-1} d^{-1}, \quad c \rightarrow 1, \\
& b \rightarrow d c d^{-1} c^{-1}, \quad d \rightarrow 1,
\end{aligned}
$$

because $\phi(a b)=1$. These are two expressions of the same homomorphism on $\pi$, but they induce different homomorphisms, $\phi_{F}: F \rightarrow F$.

Using the first choice of $\phi_{F}$ given above, we see that

$$
\phi_{F}(R)=c d c^{-1} d^{-1} a b a^{-1} b^{-1} d c d^{-1} c^{-1} b a b^{-1} a^{-1} \in F .
$$

Thus

$$
\phi_{F}(R)=\left(b a b^{-1} a^{-1}\right) R\left(b a b^{-1} a^{-1}\right)^{-1} R^{-1} \in F,
$$

and we have $A=b a b^{-1} a^{-1}-1$. The diagonal entries of $J\left(\phi_{F}\right)$ can be calculated to give us the generalized Lefschetz number

$$
L(f, \widetilde{f})=\rho\left(1-a+a b a^{-1} b^{-1}+b a b^{-1} a^{-1}-1\right) .
$$

Because for any $x \in \pi$ we have $\rho(x)=\rho(\phi(x))$, we see that

$$
\rho(a)=\rho\left(c d c^{-1} d^{-1}\right)=\rho\left(b a b^{-1} a^{-1}\right)=\rho(1),
$$

and $\rho\left(a b a^{-1} b^{-1}\right)=\rho(1)$. Hence all the orbits appearing in $L(f, \widetilde{f})$ are equal to $\rho(1)$, so $L(f, \tilde{f})$ reduces to $\rho(1)$, and $N(f)=1$. This is much more difficult than the argument that follows using the second choice for $\phi_{F}$.

Using the second choice for $\phi_{F}$, we see that $\phi_{F}(R)=1 \in F$ and $A=0$. The diagonal entries of $J\left(\phi_{F}\right)$ are also zero. Thus we very quickly see that $L(f, \widetilde{f})=$ $\rho(1)$, and no distinguishing of orbits is required.

Example 4.3. This example is similar to Example 4.1. The homomorphism $\phi$ is an isomorphism. Let $n \geq 2$ and let $\phi$ be given by

$$
\begin{array}{ll}
a \rightarrow a^{-n+1} b^{-1}, & c \rightarrow c \\
b \rightarrow b a^{n}, & d \rightarrow d .
\end{array}
$$

We claim that $N(f)=n$.

In [8], Fadell and Husseini computed $N(f)$ for the special case when $n=2$. We have $\phi_{F}(R)=R$, so $A=1$. It follows that $L(f, \widetilde{f})$ is given by $\rho\left(-2-\frac{\partial a^{-n+1}}{\partial a}\right)$ $=\rho\left(-2-\sum_{i=1}^{n-1} a^{-i}\right)$. As in Example 4.1, it can be shown that in the abelianization of $\pi, \bar{\pi}$, the orbits of the induced action that are associated with the elements $\overline{1}, \overline{a^{-1}}, \ldots, \overline{a^{-n+1}}$ are all distinct. Thus the orbits of $1, a^{-1}, \ldots, a^{-n+1}$ are all distinct, and $N(f)=n$.

Example 4.4. Once again, the homomorphism $\phi$ is an isomorphism. Let $n \geq 2$ and let $\phi$ be given by

$$
\begin{aligned}
& a \rightarrow c^{-n+1} d^{-1}, \quad c \rightarrow a, \\
& b \rightarrow d c^{n},
\end{aligned}
$$


We have $\phi_{F}(R)=c d c^{-1} d^{-1} a b a^{-1} b^{-1}$, so $A=b a b^{-1} a^{-1}$. It follows that $L(f, \widetilde{f})$ is given by $\rho\left(1+b a b^{-1} a^{-1}\right)$. Thus $N(f)$ is either 1 or 2 . We have not yet found a method for proving that these classes are distinct. Here is a example of the need for more techniques to distinguish Reidemeister orbits. The computer algebra system Magma, [2], has been used to test all words $w$ in $\pi$ of length less than eight to determine whether $\phi(w) w^{-1}$ equals $b a b^{-1} a^{-1}$. According to Magma, no solution of length less than eight exists when $n=3$. We have not yet found a proof that there is no solution of any word-length for any $n$.

Example 4.5. In the paragraph after this example we discuss a different method, from [25], by which this example can be done.

Let $n \geq 1, m \geq 1$, and let $\phi$ be given by

$$
\begin{aligned}
& a \rightarrow a^{-n} b^{-1}, \quad c \rightarrow c^{-m} d^{-1}, \\
& b \rightarrow b a^{n}, \quad d \rightarrow d c^{m} .
\end{aligned}
$$

We claim that $N(f)=n+m-1$.

Because $\phi_{F}(R)=1$ in $F$, we have $A=0$. Thus

$$
L(f, \tilde{f})=\rho\left(-1+\sum_{i=1}^{n} a^{-i}+\sum_{j=1}^{m} c^{-j}\right) .
$$

As in Example 4.1, we abelianize and discover that all the orbits appearing in the given expression for $L(f, \widetilde{f})$ are distinct with the possible exceptions of $\rho(1), \rho\left(a^{-n}\right)$, and $\rho\left(c^{-m}\right)$. In other words, the abelianization tells us that these three classes are not necessarily distinct, but each of them is distinct from $\rho\left(a^{-i}\right)$ for $1 \leq i \leq n-1$ and from $\rho\left(c^{-j}\right)$ for $1 \leq j \leq m-1$. We note that

$$
\begin{aligned}
\rho(1) & =\rho\left(\phi(a) a^{-1}\right) \rho\left(a^{-n} b^{-1} a^{-1}\right) \\
& =\rho\left(\phi\left(a^{-n}\right) \phi\left(b^{-1} a^{-1}\right)\right)=\rho\left(\phi\left(a^{-n}\right)\right)=\rho\left(a^{-n}\right),
\end{aligned}
$$

and thus there are $n+m-1$ distinct classes in the reduced form of $L(f, \widetilde{f})$.

Examples that cannot be done with any other method. Let $\phi$ be given by

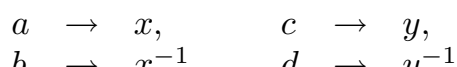

for $x$ and $y$ any elements of $\pi$. Any map $f$ inducing $\phi$ factors through the figureeight, and $\phi$ induces a homomorphism on the fundamental group of the figureeight. Wagner presents an algorithm in [25] for calculating $N(f)$ for maps on the figure-eight provided that the map satisfies a property called having remnant. Example 4.5 does have remnant, so Wagner's algorithm could have been used. The following examples do not have remnant. Thus the Fadell-Husseini method is the only known method for calculating $N(f)$ for these examples.

Example 4.6. Consider $\phi$ given by

$$
\begin{aligned}
& a \rightarrow b a^{-1} b, \quad c \rightarrow b^{2}, \\
& b \rightarrow b^{2}, \quad d \rightarrow b a^{-1} b .
\end{aligned}
$$

We have $A=0$ and $L(f, \widetilde{f})=\rho\left(b a^{-1}-b\right)$. By abelianization we see that the two classes are distinct. Thus $N(f)=2$. 
Example 4.7. Consider $\phi$ with $n \geq 1$ given by

$$
\begin{aligned}
& a \rightarrow a^{n} c^{2} a, \quad c \rightarrow b^{2} a c d, \\
& b \rightarrow a^{-1} c^{-2} a^{-n}, \quad d \rightarrow d^{-1} c^{-1} a^{-1} b^{-2} .
\end{aligned}
$$

We have $A=0$ and

$$
L(f, \tilde{f})=\rho\left(-\sum_{i=1}^{n-1} a^{i}-a^{n} c^{2}-b^{2} a+d^{-1}\right) .
$$

Note that

$$
\rho\left(d^{-1}\right)=\rho\left(\phi\left(d^{-1}\right)\right)=\rho\left(b^{2} a c d\right)=\rho\left(\phi\left(b^{2} a\right)\right)=\rho\left(b^{2} a\right) .
$$

Thus

$$
L(f, \tilde{f})=\rho\left(-\sum_{i=1}^{n-1} a^{i}-a c^{2}\right),
$$

and by abelianization we see that the classes listed are all distinct. Thus $N(f)=n$.

\section{The Klein Bottle}

The Nielsen numbers for any self-map on the Klein bottle were calculated using geometric arguments by Halpern in [10]. We use the method of Fadell and Husseini [8] to compute the Nielsen numbers algebraically. These algebraic arguments for the Klein bottle first appeared in Hart's dissertation [11].

For the Klein bottle, the Nielsen number $N(f)$ provides precise geometric information because it equals $\operatorname{Min}(f)$. A proof of this fact was circulated in preprint form by Halpern [10] in 1978 but never published. We supply a sketch of his proof at the end of this section.

Let $K$ be the Klein bottle. The fundamental group of $K$ is

$$
\pi=\left\langle a, b: a b a b^{-1}\right\rangle \text {. }
$$

The following proposition appears in [10], and we repeat it here.

Proposition 5.1 (Halpern). Let $\pi=\left\langle a, b: a b a b^{-1}=1\right\rangle$. Let $u, v, w \in \mathbb{Z}$ so that whenever $w$ is even we have $u=0$. Then there is a homomorphism $\phi: \pi \rightarrow \pi$ satisfying $\phi(a)=a^{u}$ and $\phi(b)=a^{v} b^{w}$, and every homomorphism from $\pi$ to $\pi$ is of this form.

Proof (Halpern). Every element of $\pi$ can be written in the form $a^{i} b^{j}$ for some $i, j \in \mathbb{Z}$, using the relation $b^{k} a^{m}=a^{(-1)^{k} m} b^{k}$. Thus for any homomorphism $\phi$ : $\pi \rightarrow \pi$ we have $\phi(a)=a^{u} b^{z}$ and $\phi(b)=a^{v} b^{w}$ for some $u, v, w, z \in \mathbb{Z}$. We must have $\phi\left(a b a b^{-1}\right)=1$, so we have

$$
1=\phi\left(a b a b^{-1}\right)=a^{u} b^{z} a^{v} b^{w} a^{u} b^{z} b^{-w} a^{-v}=a^{\alpha} b^{\beta},
$$

with $\alpha=u+(-1)^{z} v+(-1)^{z+w} u+(-1)^{2 z}(-v)$ and $\beta=2 z$. Thus $\beta=2 z=0$, and $\alpha=\left(1+(-1)^{w}\right) u=0$.

To calculate the Nielsen number algebraically, we first note that $\nabla R=(1+$ $a, a-1)$ and

$$
J\left(\phi_{F}\right)=\left[\begin{array}{cc}
\frac{\partial a^{u}}{\partial a} & 0 \\
\frac{\partial a^{v}}{\partial a} & a^{v} \frac{\partial b^{w}}{\partial b}
\end{array}\right] .
$$


The next step is to find $A$, the trace in dimension two. The formula in Section 3 is not useful here. It can be extremely difficult to determine the correct form for the element $\phi_{F}(R)$. On the other hand, $\pi$ is reasonable in this case in the sense that any element of $\pi$ can be written in the form $a^{i} b^{j}$ for some integers $i$ and $j$. We use factoring to find $A$.

Using the second coordinate of $\nabla R$, we see that $A$ must satisfy

$$
A(a-1)=\left(a^{u}-1\right) a^{v} \frac{\partial b^{w}}{\partial b}
$$

The following proposition states that $A$ is the unique element of $\mathbb{Z}[\pi]$ satisfying equation (2). Thus we need to examine only the second coordinate of equation (1) to find $A$. Any element that is a solution to equation (2) must be the unique solution to equation (1).

Proposition 5.2. Any element $B$ of $\mathbb{Z}[\pi]$ satisfying

$$
B(a-1)=\left(a^{u}-1\right) a^{v} \frac{\partial b^{w}}{\partial b}
$$

also satisfies the equation $B(\nabla R)=\phi(\nabla R) J\left(\phi_{F}\right)$. But $A$ is the unique element satisfying the latter equation. Thus $B=A$.

Proof. Assume $B \in \mathbb{Z}[\pi]$ with $B(a-1)=\left(a^{u}-1\right) a^{v} \frac{\partial b^{w}}{\partial b}$. Then $A(a-1)=B(a-1)$ and $(A-B)(a-1)=0$. Let $C=A-B$. Note that for any $k, C a^{k}=C$. Assume $C \neq 0$. Then $C=\sum_{i=1}^{n} \lambda_{i} a^{\alpha_{i}} b^{\beta_{i}}$ with $n \geq 1, \lambda_{i} \neq 0, \forall i=1, \ldots, n$ and $\left(\alpha_{i}, \beta_{i}\right) \neq\left(\alpha_{j}, \beta_{j}\right)$ for $j \neq i$. $C a=\sum_{i=1}^{n} \lambda_{i} a^{\alpha_{i}} b^{\beta_{i}}=\sum_{i=1}^{n} \lambda_{i} a^{\gamma_{i}} b^{\beta_{i}}$ with $\gamma_{i}=\alpha_{i}+(-1)^{\beta_{i}}$. Because $C a=C, \alpha_{i}+(-1)^{\beta_{i}}=\alpha_{j}$ and $\beta_{i}=\beta_{j}$ for some $j \neq i, 1 \leq j \leq n$.

Consider the pair $\left(\alpha_{1}, \beta_{1}\right)$. Without loss of generality, we may assume the terms of the sum are ordered so that $\alpha_{2}=\alpha_{1}+(-1)^{\beta_{1}}$ and $\beta_{2}=\beta_{1}$. For any $\ell \geq 1$, $C a^{\ell}=C$. Thus $C=\sum_{i=1}^{n} \lambda_{i} a^{\alpha_{i}} b^{\beta_{i}} a^{\ell}=\sum_{i=1}^{n} \lambda_{i} a^{\mu_{i}} b^{\beta_{i}}$ for $\mu_{i}=\alpha_{i}+\ell(-1)^{\beta_{i}}$. The pair $\left(\mu_{i}, \beta_{i}\right)$ must equal $\left(\alpha_{j}, \beta_{j}\right)$ for some $j \neq i, 1 \leq j \leq n$. Thus without loss of generality, we may assume the terms of the sum are ordered so that

$$
\alpha_{\ell+1}=\alpha_{1}+\ell(-1)^{\beta_{1}} \text {, and } \beta_{\ell+1}=\beta_{1}, 1 \leq \ell \leq n-1 .
$$

But $C a^{n}=C$ also. Thus there exists $j$ with $1<j \leq n$ such that $\alpha_{j}=\alpha_{1}+n(-1)^{\beta_{1} a}$. By equation (3), $\alpha_{j}=\alpha_{1}+(j-1)(-1)^{\beta_{1} a}$. Thus $j=n+1$. But $C$ has exactly $n$ terms. This is a contradiction, so the assumption that $C \neq 0$ is false. Finally, $C=A-B=0$, and $A=B$.

To find $A$, we need only solve $A(a-1)=a^{v}\left(a^{u}-1\right) \frac{\partial b^{w}}{\partial b}$. Let $\mathcal{E}\left(b^{w}\right)$ and $\mathcal{O}\left(b^{w}\right)$ be the sum of the even and odd powers of $b$ in $\frac{\partial b^{w}}{\partial b}$, respectively, so that $\mathcal{E}\left(b^{w}\right)+\mathcal{O}\left(b^{w}\right)=\frac{\partial b^{w}}{\partial b}$.

Proposition 5.3. The expression $A=a^{v} \frac{\partial a^{u}}{\partial a} \mathcal{E}\left(b^{w}\right)+a^{v}\left(1-\frac{\partial a^{u+1}}{\partial a}\right) \mathcal{O}\left(b^{w}\right)$ satisfies equation (2). 
Proof. Case 1. $u=0$. Because $\frac{\partial 1}{\partial a}=0, A(1-a)=a^{v}(1-1) \frac{\partial b^{w}}{\partial b}=0$. Thus $A a=A$. As in the proof of Proposition 5.2, this forces $A=0$. But for $u=0$,

$$
a^{v} \frac{\partial a^{u}}{\partial a} \mathcal{E}\left(b^{w}\right)+a^{v}\left(1-\frac{\partial a^{u+1}}{\partial a}\right) \mathcal{O}\left(b^{w}\right)=0=A .
$$

Case 2. $u \neq 0$. Note that $a^{u}-1$ factors in two ways. For $u>0$, we have

$$
a^{u}-1=\left(1+a+a^{2}+\cdots+a^{u-1}\right)(a-1)=\frac{\partial a^{u}}{\partial a}(a-1),
$$

and

$$
a^{u}-1=-\left(a+a^{2}+\cdots+a^{u}\right)\left(a^{-1}-1\right)=\left(1-\frac{\partial a^{u+1}}{\partial a}\right)\left(a^{-1}-1\right) .
$$

For $u<0$, we have

and

$$
a^{u}-1=-\left(a^{-1}+a^{-2}+\cdots+a^{u}\right)(a-1)=\frac{\partial a^{u}}{\partial a}(a-1),
$$

$$
\begin{aligned}
a^{u}-1 & =\left(1+a^{-1}+a^{-2}+\cdots+a^{u+1}\right)\left(a^{-1}-1\right) \\
& =\left(1-\frac{\partial a^{u+1}}{\partial a}\right)\left(a^{-1}-1\right) .
\end{aligned}
$$

Thus for all $u \neq 0$ we have

$$
\begin{aligned}
A(a-1) & =a^{v}\left(a^{u}-1\right) \frac{\partial b^{w}}{\partial b} \\
& =a^{v} \frac{\partial a^{u}}{\partial a}(a-1) \mathcal{E}\left(b^{w}\right)+a^{v}\left(1-\frac{\partial a^{u+1}}{\partial a}\right)\left(a^{-1}-1\right) \mathcal{O}\left(b^{w}\right) .
\end{aligned}
$$

Recall that $a \mathcal{E}\left(b^{w}\right)=\mathcal{E}\left(b^{w}\right) a$ and $a^{-1} \mathcal{O}\left(b^{w}\right)=\mathcal{O}\left(b^{w}\right) a$. Thus

$$
A(a-1)=\left[a^{v} \frac{\partial a^{u}}{\partial a} \mathcal{E}\left(b^{w}\right)+a^{v}\left(1-\frac{\partial a^{u+1}}{\partial a}\right) \mathcal{O}\left(b^{w}\right)\right](a-1),
$$

and for $u>0$,

$$
A=a^{v} \frac{\partial a^{u}}{\partial a} \mathcal{E}\left(b^{w}\right)+a^{v}\left(1-\frac{\partial a^{u+1}}{\partial a}\right) \mathcal{O}\left(b^{w}\right) .
$$

$L(f, \widetilde{f})$ for self-maps on the Klein bottle. The Fadell-Husseini formula for $L(f, \widetilde{f})$ applied to $f: K \rightarrow K$ is

$$
\begin{aligned}
L(f, \widetilde{f})= & \rho\left(1-\operatorname{tr} J\left(\phi_{F}\right)+A\right) \\
= & \rho\left(1-\frac{\partial a^{u}}{\partial a}-a^{v} \frac{\partial b^{w}}{\partial b}+a^{v} \frac{\partial a^{u}}{\partial a} \mathcal{E}\left(b^{w}\right)+a^{v}\left(1-\frac{\partial a^{u+1}}{\partial a}\right) \mathcal{O}\left(b^{w}\right)\right) \\
=\rho & \left(1-\frac{\partial a^{u}}{\partial a}-a^{v} \mathcal{E}\left(b^{w}\right)-a^{v} \mathcal{O}\left(b^{w}\right)\right. \\
& \left.+a^{v} \frac{\partial a^{u}}{\partial a} \mathcal{E}\left(b^{w}\right)+a^{v}\left(1-\frac{\partial a^{u+1}}{\partial a}\right) \mathcal{O}\left(b^{w}\right)\right) .
\end{aligned}
$$

Thus

$$
L(f, \tilde{f})=\rho\left(1-\frac{\partial a^{u}}{\partial a}+a^{v}\left(\frac{\partial a^{u}}{\partial a}-1\right) \mathcal{E}\left(b^{w}\right)-a^{v} \frac{\partial a^{u+1}}{\partial a} \mathcal{O}\left(b^{w}\right)\right) .
$$

Next we must express the generalized Lefschetz number of $f$ in reduced form with each Reidemeister orbit appearing at most once. 
Distinguishing Reidemeister orbits. Given two elements of $\pi$ that appear in the sum $L(f, \tilde{f})$, we determine whether they are in the same Reidemeister orbit.

Proposition 5.4. Given two elements $a^{i} b^{j}, a^{r} b^{s} \in \pi$, whenever $j \not \equiv s \bmod (w-1)$, we have $\rho\left(a^{i} b^{j}\right) \neq \rho\left(a^{r} b^{s}\right)$.

Proof. Let $N$ be the normal closure of the subgroup generated by $a$ and $b^{w-1}$ in $\pi$. Then $\bar{\pi}=\pi / N$ is isomorphic to $\mathbb{Z}_{|w-1|}$ with $\mathbb{Z}_{0}=\mathbb{Z}$ and $\mathbb{Z}_{1}$ the trivial group. The generator of $\bar{\pi}$ is $\bar{b}$. The subgroup $N$ of $\pi$ is generated by $\left\{c a c^{-1}, c b^{w-1} c^{-1}: c \in \pi\right\}$. We have $\phi(N) \subseteq(N)$. Thus $\bar{\phi}: \bar{\pi} \rightarrow \bar{\pi}$ is well-defined, and $\bar{\phi}$ is the identity homomorphism. Two elements of $\bar{\pi}$ are in the same orbit of the action induced by the Reidemeister action iff they are conjugate in $\bar{\pi}$. Therefore each orbit is a singleton in $\bar{\pi}$. Thus

$$
\rho\left(a^{i} b^{j}\right)=\rho\left(a^{r} b^{s}\right) \Rightarrow \bar{b}^{j}=\bar{b}^{s} \in \bar{\pi} \Rightarrow j \equiv s \bmod (w-1) .
$$

The reduced form of $L(f, \tilde{f})$.

Proposition 5.5. Let $a^{i} b^{j}$ and $a^{r} b^{s}$ be two of the elements of $\pi$ that appear in the sum $L(f, \widetilde{f})$ in equation (4). Then for $w>0$,

$$
\rho\left(a^{i} b^{j}\right)=\rho\left(a^{r} b^{s}\right) \Rightarrow j=s \quad \text { or }(j, s)=(0, w-1) \text { or }(j, s)=(w-1,0) .
$$

For $w<0$,

$$
\rho\left(a^{i} b^{j}\right)=\rho\left(a^{r} b^{s}\right) \Rightarrow j=s .
$$

Proof. The sum $L(f, \widetilde{f})$ as in equation (4) contains the following powers of $b$ :

$$
\begin{aligned}
& \text { For } w>0, b^{0}, b^{1}, b^{2}, \ldots, b^{w-1} . \\
& \text { For } w<0, b^{0}, b^{-1}, b^{-2}, \ldots, b^{w} .
\end{aligned}
$$

By equation (5), the exponents of $b$ to consider are those that are equivalent modulo $w-1$. The only such exponents appearing in $L(f, \widetilde{f})$ are 0 and $w-1$ for $w>0$. Thus the two elements of $\pi$ could possibly be in the same orbit only if $j=s$ or, for $w>0$, if $j=0$ and $s=w-1$.

The following lemma will be helpful in the proof of the theorem.

Lemma 5.6. For $w \neq 1, w$ odd, and $i, j, r \in \mathbb{Z}$, we have when $j$ is even

$$
\rho\left(a^{i} b^{j}\right)=\rho\left(a^{r} b^{j}\right) \Leftrightarrow r \equiv i \bmod (u-1),
$$

and when $j$ is odd

$$
\rho\left(a^{i} b^{j}\right)=\rho\left(a^{r} b^{j}\right) \Leftrightarrow r \equiv i \bmod (u+1) .
$$

Proof. If $\rho\left(a^{i} b^{j}\right)=\rho\left(a^{r} b^{j}\right)$, there exist $x, y \in \mathbb{Z}$ such that

$$
a^{r} b^{j}=\phi\left(a^{x} b^{y}\right)\left(a^{i} b^{j}\right)\left(a^{x} b^{y}\right)^{-1} .
$$

This forces $j=j+y(w-1)$, and thus $y=0$. The equation above reduces to

$$
a^{r} b^{j}=\phi\left(a^{x}\right)\left(a^{i} b^{j}\right)\left(a^{x}\right)^{-1}
$$

which forces $r \equiv i \bmod (u-1)$ for even values of $j$ and $r \equiv i \bmod (u+1)$ for odd values of $j$.

To prove the other implication for $j$ even, assume $r=i+p(u-1)$ for some $p \in \mathbb{Z}$. Let $a^{p}$ act on $a^{i} b^{j}$ via the Reidemeister action. We see that $\rho\left(a^{i} b^{j}\right)=\rho\left(a^{r} b^{j}\right)$. For odd values of $j$, assume $r=i+p(u+1)$ for some $p \in \mathbb{Z}$. Let $a^{p}$ act on $a^{i} b^{j}$. 
Theorem 5.7 (Halpern). The Nielsen number for a self-map $f$ on the Klein bottle is given in terms of a homomorphism $\phi: \pi \rightarrow \pi$ that is induced by a cellular approximation to $f$ and that satisfies $\phi(a)=a^{u}$ and $\phi(b)=a^{v} b^{w}$. We have

$$
N(f)= \begin{cases}|u(w-1)| & \text { for } u \neq 0 \\ |w-1| & \text { for } u=0\end{cases}
$$

Also,

$$
L(f)= \begin{cases}-\operatorname{sgn}(w)|w-1|, & w \neq 0 \\ 1, & w=0\end{cases}
$$

Proof. Part 1. $w=0$. Whenever $w=0$, we have $u=0$ and $L(f, \tilde{f})=\rho(1)$. Thus $N(f)=1=|w-1|$.

Part 2. $w \neq 0$ and $w$ even. Whenever $w$ is even, $u=0$. Thus for $w$ even

$$
L(f, \widetilde{f})=1-a^{v} \mathcal{E}\left(b^{w}\right)-a^{v} \mathcal{O}\left(b^{w}\right)=1-a^{v} \frac{\partial b^{w}}{\partial b} .
$$

By Proposition 5.5, the only terms in this sum that could be in the same Reidemeister orbit are $1, a^{v}$, and $a^{v} b^{w-1}$ if $w>0$. For $w<0$, none of the terms are in the same orbit. For $w>0$, let $b$ act on 1 via the Reidemeister action. Then

$$
\phi(b)(1) b^{-1}=a^{v} b^{w-1} .
$$

Thus for $w>0$ and $w$ even, $\rho(1)=\rho\left(a^{v} b^{w-1}\right)$, and $L(f, \widetilde{f})$ is

$$
L(f, \tilde{f})= \begin{cases}-\rho\left(a^{v}\right)-\rho\left(a^{v} b\right)-\cdots-\rho\left(a^{v} b^{w-2}\right), & w>0 \text { and } w \text { even } \\ \rho(1), & w=0, \\ \rho(1)+\rho\left(a^{v} b^{-1}\right)+\cdots+\rho\left(a^{v} b^{w}\right), & w<0 \text { and } w \text { even }\end{cases}
$$

The form for $L(f, \widetilde{f})$ given here is reduced, because all the orbits shown are distinct by Proposition 5.5. Thus $N(f)$ is $|w-1|$ for $w$ even.

Part 3. $w$ odd. Let

$$
\sigma=1-\frac{\partial a^{u}}{\partial a}+a^{v}\left(\frac{\partial a^{u}}{\partial a}-1\right) .
$$

By substituting $\sigma$ into equation (4), we find that

$$
L(f, \tilde{f})=\rho\left(\sigma+a^{v}\left(\frac{\partial a^{u}}{\partial a}-1\right)\left(\mathcal{E}\left(b^{w}\right)-1\right)-a^{v} \frac{\partial a^{u+1}}{\partial a} \mathcal{O}\left(b^{w}\right)\right) .
$$

Step 1. $\rho(\sigma)=0$. To prove that $\rho(\sigma)=0$, we first note that for $i, r \in \mathbb{Z}$ with $r \equiv i \bmod (u-1), \rho\left(a^{r}\right)=\rho\left(a^{i}\right)$. (Let $c \in \mathbb{Z}$, so that $r=i+c(u-1)$, and let $a^{c}$ act on $a^{i}$ via the Reidemeister action.)

Case 1. $u>0$. Note that $\sigma=-\sum_{n=1}^{u-1} a^{n}+\sum_{n=1}^{u-1} a^{v+n}$. Let $q \in \mathbb{Z}$ be such that $v \equiv q \bmod (u-1)$ and $0<q \leq u-1$. Then for any $x \in \mathbb{Z}, \rho\left(a^{v+x}\right)=\rho\left(a^{q+x}\right)$. 
Thus

$$
\begin{aligned}
\rho(\sigma) & =-\sum_{n=1}^{u-1} \rho\left(a^{n}\right)+\sum_{n=1}^{u-1} \rho\left(a^{q+n}\right) \\
& =-\sum_{n=1}^{q} \rho\left(a^{n}\right)-\sum_{n=q+1}^{u-1} \rho\left(a^{n}\right)+\sum_{n=1}^{u-1-q} \rho\left(a^{q+n}\right)+\sum_{n=u-q}^{u-1} \rho\left(a^{q+n}\right) \\
& =-\sum_{n=1}^{q} \rho\left(a^{n}\right)-\sum_{n=q+1}^{u-1} \rho\left(a^{n}\right)+\sum_{n=q+1}^{u-1} \rho\left(a^{n}\right)+\sum_{n=u-q}^{u-1} \rho\left(a^{q+n}\right) \\
& =-\sum_{n=1}^{q} \rho\left(a^{n}\right)+\sum_{n=u-q}^{u-1} \rho\left(a^{q+n}\right) \\
& =-\sum_{n=1}^{q} \rho\left(a^{n}\right)+\sum_{n=1}^{q} \rho\left(a^{n+u-1}\right) \\
& =0 .
\end{aligned}
$$

Case 2. $u=0$. In this case, $\sigma=1-a^{v}=a^{0}-a^{v}$. Any two integers are equivalent modulo -1 , so $0 \equiv v \bmod -1$. Thus $\rho(\sigma)=\rho(1)-\rho\left(a^{v}\right)=0$.

Case 3. $u<0$. Let $q \in \mathbb{Z}$ such that $u \leq q \leq 0$ and $q \equiv v \bmod (u-1)$. For any $x \in \mathbb{Z}, \rho\left(a^{v+x}\right)=\rho\left(a^{q+x}\right)$. For $u<0, \sigma=\sum_{n=0}^{|u|} a^{-n}-\sum_{n=0}^{|u|} a^{v-n}$. Therefore

$$
\begin{aligned}
\rho(\sigma) & =\sum_{n=0}^{|q|-1} \rho\left(a^{-n}\right)+\sum_{n=|q|}^{|u|} \rho\left(a^{-n}\right)-\sum_{n=0}^{q+|u|} \rho\left(a^{q-n}\right)-\sum_{n=q+|u|+1}^{|u|} \rho\left(a^{q-n}\right) \\
& =\sum_{n=0}^{|q|-1} \rho\left(a^{-n}\right)+\sum_{n=|q|}^{|u|} \rho\left(a^{-n}\right)-\sum_{n=|q|}^{|u|} \rho\left(a^{-n}\right)-\sum_{n=0}^{|q|-1} \rho\left(a^{u-1-n}\right) \\
& =\sum_{n=0}^{|q|-1} \rho\left(a^{-n}\right)-\sum_{n=0}^{|q|-1} \rho\left(a^{u-1-n}\right) \\
& =0 .
\end{aligned}
$$

Thus $\rho(\sigma)=0$, and Step 1 is completed.

Step 2. The proof for $w=1$. For $w=1, L(f, \tilde{f})=\rho(\sigma)=0$. There are no terms with coefficient different from zero, so $N(f)=0=|u(w-1)|$.

Step 3. The proof for $w \neq 1, w$ odd. We have that

$$
L(f, \tilde{f})=\rho\left(a^{v}\left(\frac{\partial a^{u}}{\partial a}-1\right)\left(\mathcal{E}\left(b^{w}\right)-1\right)-a^{v} \frac{\partial a^{u+1}}{\partial a} \mathcal{O}\left(b^{w}\right)\right) .
$$

Let $B=a^{v}\left(\frac{\partial a^{u}}{\partial a}-1\right)\left(\mathcal{E}\left(b^{w}\right)-1\right)$, and let $C=a^{v} \frac{\partial a^{u+1}}{\partial a} \mathcal{O}\left(b^{w}\right)$. Then $L(f, \tilde{f})=$ $\rho(B-C)$.

The sum $B$ can be written uniquely (up to the ordering of the terms) as

$$
B=\sum_{n=1}^{\ell} \lambda_{n} a^{i_{n}} b^{j_{n}}
$$


with $\left(i_{n}, j_{n}\right) \neq\left(i_{m}, j_{m}\right)$ for all $n \neq m$, with $\lambda_{n} \in \mathbb{Z}$, and with $\lambda_{n} \neq 0$ for $n=$ $1, \ldots, \ell$. Note that for $w>0, j_{n}$ is never zero. Thus for any $w$, Proposition 5.5 implies that for $1 \leq n \leq \ell$ and $1 \leq m \leq \ell$,

$$
\rho\left(a^{i_{n}} b^{j_{n}}\right)=\rho\left(a^{i_{m}} b^{j_{m}}\right) \Rightarrow j_{n}=j_{m} .
$$

Given two elements of $\pi$ appearing in equation (7) with the same power of $b$, are they in the same Reidemeister orbit?

In equation (7), the possible values for $i_{n}$ are as follows:

$$
\begin{aligned}
& \text { For } u>0, \quad i_{n}=v+1, v+2, \ldots, v+u-1 . \\
& \text { For } u=0, \quad i_{n}=v \\
& \text { For } u<0, \quad i_{n}=v, v-1, \ldots, v+u
\end{aligned}
$$

For any value of $u$, none of the values for $i_{n}$ are equivalent modulo $u-1$. Therefore, using Lemma 5.6, the reduced form of $\rho(B)$ is

$$
\rho(B)=\rho\left(a^{v}\left(\frac{\partial a^{u}}{\partial a}-1\right)\left(\mathcal{E}\left(b^{w}\right)-1\right)\right) .
$$

Next we consider $C=a^{v} \frac{\partial a^{u+1}}{\partial a} \mathcal{O}\left(b^{w}\right)$. Just as in the calculations for $B, C$ can be written uniquely (up to the ordering of the terms) as

$$
C=\sum_{n=1}^{\ell} \lambda_{n} a^{i_{n}} b^{j_{n}}
$$

with $\left(i_{n}, j_{n}\right) \neq\left(i_{m}, j_{m}\right)$ for all $n \neq m$, with $\lambda_{n} \in \mathbb{Z}$ and with $\lambda_{n} \neq 0$ for $n=$ $1, \ldots, \ell$. Note that $j_{n}$ is never zero. Thus for any $w$, Proposition 5.5 implies that for $1 \leq n \leq \ell$ and $1 \leq m \leq \ell$,

$$
\rho\left(a^{i_{n}} b^{j_{n}}\right)=\rho\left(a^{i_{m}} b^{j_{m}}\right) \Rightarrow j_{n}=j_{m} .
$$

Given two elements of $\pi$ appearing in equation (8) with the same power of $b$, are they in the same Reidemeister orbit?

Using Lemma 5.6, we see that the possible values for $i_{n}$ in equation (8) are as follows:

$$
\begin{aligned}
& \text { For } u>0, \quad i_{n}=v, v+1, \ldots, v+u \\
& \text { For } u=0, \quad i_{n}=v \\
& \text { For } u<0, \quad i_{n}=v-1, v-2, \ldots, v+u+1 .
\end{aligned}
$$

For any value of $u$, none of the values for $i_{n}$ are equivalent modulo $u+1$. Therefore, the reduced form of $\rho(C)$ is

$$
\rho(C)=\rho\left[a^{v} \frac{\partial a^{u+1}}{\partial a} \mathcal{O}\left(b^{w}\right)\right] .
$$

By Proposition 5.5, no element of $\pi$ appearing in the sum $B$ is in the same Reidemeister orbit as an element of $\pi$ appearing in $C$. Therefore, $\rho(B-C)=$ $L(f, \widetilde{f})$ is in reduced form. For example, if $u>0, w>1$, and $w$ is odd, we have

$$
\sum_{\ell=1}^{\frac{w-1}{2}} \sum_{k=1}^{u-1} \rho\left(a^{v+k} b^{2 \ell}\right)-\sum_{\ell=0}^{\frac{w-3}{2}} \sum_{k=0}^{u} \rho\left(a^{v+k} b^{2 \ell+1}\right) .
$$


The Nielsen number of $f, N(f)$, is the total number of terms in $\rho(B-C)$ with coefficient different from zero. Whenever $w$ is odd, a simple counting of terms gives

$$
N(f)= \begin{cases}|u(w-1)|, & u \neq 0 \\ |w-1|, & u=0 .\end{cases}
$$

The Lefschetz number of $f, L(f)$, is the sum of the coefficients of $\rho(B-C)$. Thus $L(f)=-\operatorname{sgn}(w)|w-1|$ for any odd value of $w$.

The Wecken property for the Klein bottle. Here we include the proof from the unpublished [10] that the Klein bottle is Wecken.

Theorem 5.8 (Halpern). The Klein bottle $K$ is Wecken. That is, $N(f)=\operatorname{Min}(f)$ for any map $f: K \rightarrow K$.

Sketch of the proof. Halpern classifies the homotopy classes of maps from $K$ to $K$, using the homotopy extension property and inner automorphisms, in terms of $u, v$, and $w$ as in Proposition 5.1. For any map $f: K \rightarrow K$, he proves in Theorem 2.7 of [10] that $f$ is homotopic to a map $g$ that fixes the base point of $K$ and induces a homomorphism $\phi: \pi \rightarrow \pi$ with the following special properties:

1. $u \geq 0$.

2. If $w$ is even, $u=0$.

3. $v \geq 0$.

4. If $w$ is odd, then $v=0$ or 1 .

Because every homotopy class contains a map that induces a homomorphism of this special form, these are the only homomorphisms we need to consider in this proof.

For each homomorphism $\phi$ of the form described in the previous paragraph, Halpern exhibits a map $f: K \rightarrow K$ that is homotopic to a map that fixes the base point of $K$ and induces $\phi$. The original map $f$ is such that $|\operatorname{Fix}(f)|$ can be determined geometrically to equal $N(f)$. He does this in terms of lifts $\widetilde{f}: \mathbb{R}^{2} \rightarrow \mathbb{R}^{2}$.

For each triple $(u, v, w)$, define $\widetilde{f}$ as in Table 1 for all points $(x, y) \in \mathbb{R}^{2}$. We will see that $\widetilde{f}$ covers a map $f: K \rightarrow K$ and that this map $f$ is homotopic to a map that induces $\phi$.

First, for any odd integer $w \neq 1$, define $\lambda_{w}: \mathbb{R} \rightarrow \mathbb{R}$ to be any map satisfying $\lambda_{w}(y+1)=-\lambda_{w}(y)$ for all $y \in \mathbb{R}$ and satisfying

$$
\lambda_{w}\left(\frac{j}{w-1}\right)= \begin{cases}\sqrt{2} & \text { if } j \text { is an even integer and } \frac{j}{w-1} \in[0,1), \\ 0 & \text { if } j \text { is an odd integer and } \frac{j}{w-1} \in[0,1) .\end{cases}
$$

Let the generators of $\pi$ be given in the form of covering transformations as $a(x, y)=(x+1, y)$ and $b(x, y)=(-x, y+1)$. To prove that $\widetilde{f}$ covers a map on $K$ it suffices to prove that there exist elements $c, d \in \pi$ so that $\widetilde{f} \circ a(x, y)=c \circ \widetilde{f}(x, y)$ and $\widetilde{f} \circ b(x, y)=d \circ \widetilde{f}(x, y)$. The reader can check that in Case 1 we have $c=1$ and $d=a^{v} b^{w}$. For Cases 2-7, $c=a^{u}$. For Cases 2, 4, and 5, $d=b^{w}$. Finally, for Cases 3, 6, and 7, $d=a b^{w}$.

Each $\widetilde{f}$ covers a map $f$ that is homotopic to a map $g$ that induces a homomorphism with the values of $u, v$, and $w$ given in Table ??. We prove this for Case 6 and leave the remaining cases to the reader. Let $\alpha, \beta, \gamma_{1}, \gamma_{2}$ and $\gamma_{3}$ be paths in $\mathbb{R}^{2}$ 


\section{TABLE 1}

\begin{tabular}{|l|l|l|}
\hline \hline Case 1 & $w$ even & $\widetilde{f}(x, y)=(v y, w y)$ \\
\hline Case 2 & $w=1, v=0$ & $\widetilde{f}(x, y)=(u x, y+\sqrt{2})$ \\
\hline Case 3 & $\mathrm{w}=1, \mathrm{v}=1$ & $\widetilde{f}(x, y)=\left(\frac{1}{2}+u x, y+\sqrt{2}\right)$ \\
\hline Case 4 & $w$ odd, $w \neq 1, v=0, u \neq 1$ & $\widetilde{f}(x, y)=(u x, w y)$ \\
\hline Case 5 & $w$ odd, $w \neq 1, v=0, u=1$ & $\widetilde{f}(x, y)=\left(x+\lambda_{w}(y), w y\right)$ \\
\hline Case 6 & $w$ odd, $w \neq 1, v=1, u \neq 1$ & $\widetilde{f}(x, y)=\left(\frac{1}{2}+u x, w y\right)$ \\
\hline Case 7 & $w$ odd, $w \neq 1, v=1, u=1$ & $\widetilde{f}(x, y)=\left(\frac{1}{2}+x+\lambda_{w}(y), w y\right)$ \\
\hline \hline
\end{tabular}

for $t \in I$ given by

$$
\begin{aligned}
\alpha(t) & =(t, 0), \\
\beta(t) & =(0, t), \\
\gamma_{1}(t) & =\left(\frac{1}{2}-\frac{1}{2} t, 0\right), \\
\gamma_{2}(t) & =\left(\frac{1}{2}+\frac{1}{2} t, w\right), \text { and } \\
\gamma_{3}(t) & =\left(1 \frac{1}{2}-\frac{1}{2} t, 0\right) .
\end{aligned}
$$

Then $\pi \circ \alpha \in a, \pi \circ \beta \in b$, and $\pi \circ \gamma_{1}=\pi \circ \gamma_{2}=\pi \circ \gamma_{3}$, because $w$ is odd. Set $\gamma=\pi \circ \gamma_{1}$. Then $\gamma$ is a path from $f(*)$ to $*$. Note that $\gamma_{1}^{-1}(\widetilde{f} \circ \beta) \gamma_{2} \sim \alpha \beta^{w}$ rel $\{0,1\}$, and hence $\gamma^{-1} f \circ(\pi \circ \beta) \gamma \sim(\pi \circ \alpha)(\pi \circ \beta)^{w} \operatorname{rel}\{0,1\}$. Thus $f \simeq g$ with $g_{\#}=a b^{w}$. Similarly, we note that $\gamma_{1}^{-1}(\widetilde{f} \circ \alpha) \gamma_{3} \sim \alpha \operatorname{rel}\{0,1\}$ and so $\gamma^{-1} f \circ(\pi \circ \alpha) \gamma \sim \pi \circ \alpha \operatorname{rel}\{0,1\}$. Thus $g_{\#}(a)=a^{u}$.

Next we check for each case that $f$ has exactly $N(f)$ fixed points. The condition $f(\pi(x, y))=\pi(x, y)$ is equivalent to $\widetilde{f}(x, y)=a^{i} \circ b^{j}(x, y)$ for some $(i, j) \in \mathbb{Z}^{2}$. In each of the seven cases of Definition 4.1 we obtain
CASE 1: $y=\frac{j}{w-1}$

$$
x=(-1)^{j}\left(\frac{v j}{w-1}-i\right)
$$

CASE $2: \sqrt{2}=j \quad$ (so no solutions)

CASE $3: \sqrt{2}=j \quad$ (so no solutions) 
CASE $4: y=\frac{j}{w-1} \quad x=\frac{i}{u-(-1)^{j}}$

CASE 5: If $j$ is even, then $\sqrt{2}=i$ (so no solutions).

If $j$ is odd, then $y=\frac{j}{w-1}$ and $x=\frac{i}{2}$.

CASE $6: y=\frac{j}{w-1} \quad x=\frac{i-\frac{1}{2}}{u-(-1)^{j}}$

CASE 7: If $j$ is even, then $\sqrt{2}=i-\frac{1}{2}$ (so no solutions).

If $j$ is odd, then $y=\frac{j}{w-1}$ and $x=\frac{i-\frac{1}{2}}{2}$.

To calculate the number of fixed points of $f$ in each case, we need only count the number of fixed points of $\widetilde{f}$ for which $0 \leq x<1$ and $0 \leq y<1$.

In each case it can be seen that

$$
|\operatorname{Fix}(f)|= \begin{cases}|u(w-1)| & \text { if } u \neq 0, \\ |w-1| & \text { if } u=0 .\end{cases}
$$

Thus Halpern's work proves that the Klein bottle is Wecken.

\section{REFERENCES}

[1] Bestvina, M. and Handel, M. Train tracks and surface homeomorphisms, Topology 34 (1995), 109-140. CMP 1995:5

[2] Bosma, W., Cannon, J. J., and Mathews, G. Programming with algebraic structures: Design of the Magma language, In: M. Giesbrecht (ed), Proceedings of the 1994 International Symposium on Symbolic and Algebraic Computation, Oxford, July 20-22, 1994. Association for Computing Machinery, 1994, 52-57.

[3] Brooks, R., Brown, R., Pak, J., and Taylor, D. The Nielsen number of maps of tori, Proc. AMS 52 (1975), 398-400. MR 51:11483

[4] Brouwer, L. Über die Minimalzahl der Fixpunkte bei den Klassen von eindeutigen stetigen Transformationen der Ringflächen, Math. Ann. 82 (1921), 94-96.

[5] Brown, R. F. The Lefschetz Fixed Point Theorem, Scott, Foresman and Co., 1971. MR 44:1023

[6] Brown, R. F. Wecken properties for manifolds, Nielsen Theory and Dynamical Systems, Contemp. Math., Vol. 152, 1993, 9-21. MR 94e:55002

[7] Crowell, R. and Fox, R. An Introduction to Knot Theory, Ginn and Co., 1963. MR 26:4348

[8] Fadell, E. and Husseini, S. The Nielsen number on surfaces, Contemp. Math., Vol. 21, 1983, 59-98. MR 85h:55004

[9] Franks, J. and Misiurewicz, M. Cycles for disk homeomorphisms and thick trees, Nielsen Theory and Dynamical Systems, Contemp. Math., Vol. 152, 1993 69-140. MR 95e:58133

[10] Halpern, B. Periodic points on the Klein bottle, preprint, 1978.

[11] Hart, E. An algebraic study of local Nielsen fixed point theory, Doctoral Dissertation, University of Wisconsin-Madison, 1991.

[12] Heath, P., Keppelmann, E., and Wong, P. Addition formulae for Nielsen numbers and for Nielsen type numbers of fibre preserving maps, Topology Appl. 67 (1995), 133-157. CMP 1996:4

[13] Hopf, H. Über Mindestzahlen von Fixpunkten, Math. Z., 26, 1927, 762-774.

[14] Husseini, S. Generalized Lefschetz numbers, Trans. Amer. Math. Soc. 272 (1982), 247-274. MR 83m:55004

[15] Jiang, B. Lectures on Nielsen Fixed Point Theory, Contemp. Math., Vol. 14, 1983. MR 84f:55002

[16] Jiang, B. Commutativity and Wecken properties for fixed points of surfaces and 3-manifolds, Topology Appl. 53 (1993), 221-228. MR 95c:55003 
[17] Kelly, M. The relative Nielsen number and boundary-preserving surface maps, Pacific J. Math. 161 (1993), 139-153. MR 94h:55002

[18] Kelly, M. Computing Nielsen numbers of surface homeomorphisms, Topology, to appear.

[19] Kiang, T. The Theory of Fixed Point Classes, Springer Verlag and Science Press, 1989. MR 90h:55002

[20] Lyndon, R., and Schupp, P. Combinatorial Group Theory, Springer-Verlag, 1977. MR $\mathbf{5 8 : 2 8 1 8 2}$

[21] McCord, C. Nielsen numbers and Lefschetz numbers on nilmanifolds and solvmanifolds, Pacific J. Math. 147 (1991), 153-164. MR 92a:55002

[22] McCord, C. Estimating Nielsen numbers on infrasolvmanifolds, Pacific J. Math. 154 (1992), 345-368. MR 93d:55001

[23] Nielsen, J. Über die Minimalzahl der Fixpunkte bei Abbildungstypen der Ringflächen, Math. Ann. 82 (1921), 83-93.

[24] Nielsen, J. Untersuchungen zur Topologie der geschlossenen zweiseitigen Flächen, I, Acta Math. 50 (1927), 189-358.

[25] Wagner, J. An algorithm for calculating the Nielsen number on surfaces with boundary, preprint.

Department of Mathematics, Binghamton University, Binghamton, New York 13902 6000

E-mail address: owen@math.binghamton.edu

Department of Mathematics, Hope College, Holland, Michigan 49423-9000

Current address: Department of Mathematics, Colgate University, Hamilton, New York 13346-1398

E-mail address: ehart@colgate.edu

Department of Mathematics, Dartmouth College, Hanover, New Hampshire 037553551

E-mail address: trapp@dartmouth.edu 